\title{
On the Stability of Ekman Boundary Layer Flow with Thermally Unstable Stratification
}

\author{
By Tomio Asai and Isao Nakasuji \\ Geophysical Institute and Meteorological Research Institute, Kyoto University, Kyoto \\ (Manuscript received 8 December, 1972)
}

\begin{abstract}
An investigation is made of the stability of the Ekman boundary layer flow with a thermally unstable stratification based on perturbation analysis. Three different types of the instability associated with the flow may be found, preference of which depends on Reynolds number, and Richardson number. The thermal instability, which is of gravitational origin modified by the shear flow, is dominant for $R_{i} \gtrsim 10^{-3}$, while the other two, which are of inertial origin modified by the thermal stratification, are found for $R_{i} \lesssim 10^{-3}$ where the Richardson number $R_{i}$ is taken to be positive for unstable stratification. One of the inertial instabilities may occur for low Reynolds number ranging $55 \lesssim R_{e} \lesssim 150$ and the other predominates for $R_{e} \gtrsim 150$. Stability properties of the three different types and characteristics of the associated unstable perturbations are examined in detail by means of numerical solution of the perturbation equations. It is noticed that both the types of the inertial instability are slightly influenced by thermally unstable stratification in the range of $R_{i} \lesssim 10^{-3}$.
\end{abstract}

\section{Introduction}

Experimental studies of stability of the Ekman layer flow were made by Gregory, Stuart and Walker (1955), Faller (1963, 1965), Tatro and Mollö-Christensen (1967) and others. The laboratory experiments show that the instability appears when the Reynolds number exceeds a critical value, i.e., about 125 and the waves observed form a series of horizontal roll vortices whose orientation is an angle of $14.6^{\circ}$ with respect to the geostrophic flow and whose spacing is 11.8 times as much as the characteristic depth of the boundary layer. These results are coincident with the theoretical study made by Gregory, Stuart and Walker (1955), Brown (1961), Barcilon (1965), Faller (1965) and others. Lilly (1966) and Faller and Kaylor (1966) further examined the stability of the flow for the range of smaller Reynolds numbers than 100 and showed possibility of another type of instability different from that described above when the Reynolds number exceeded 55. It was suggested that the waves would form horizontal roll vortices which were oriented at some angle clockwise from the direction of the geostrophic flow and had a preferred spacing about 20 times as much as the characte- ristic depth of the boundary layer. Lilly (1966) exhibited the unstable waves were associated with the Coriolis force and parallel component of the basic flow to the roll axes and designated them as "parallel instability". This type of instability was also confirmed by the laboratory experiments performed by Faller and Kaylor (1967) and Tatro and Mollö-Christensen (1967).

In the atmospheric boundary layer there are observed somewhat regular roll vortices (e.g., Woodcock 1940; Kuettner, 1959 and 1971; Pack, 1962; Faller, 1965; Plank, 1966; Angell, Pack and Dickson, 1968; Hanna, 1969; Brown, 1970). It may be expected that thermal stratification plays a significant role in the stability properties of the Ekman boundary layer flow in the atmosphere. Faller and Kaylor (1967) attempted a stability analysis of the stratified Ekman flow in a limited small physical domain. Quite recently Kaylor and Faller (1972) extended their previous study to the stability of the Ekman boundary layer flow with stable density stratification and elucidated a possible resonance of the Ekman flow instability with an internal gravity wave.

In this article the stability properties of the Ekman flow in an unstably stratified layer are studied in detail for $a$ wide range of physical 
parameters determining the flow characteristics.

\section{Basic equations}

The Ekman boundary layer flow may be expressed in the following form,

$$
\left.\begin{array}{l}
\bar{u}=V_{g}\left(1-e^{-z / D} \cos \frac{z}{D}\right), \\
\bar{v}=V_{g} e^{-z / D} \sin \frac{z}{D},
\end{array}\right\}
$$

where $V_{g}$ is the geostrophic flow velocity, $D$ is the characteristic depth of the boundary layer defined as

$$
D=\left(\frac{2 \nu}{f}\right)^{1 / 2}
$$

$\nu$ is the coefficient of kinematic viscosity and $f$ is the Coriolis parameter. We take the Cartesian coordinates so that $x$ measures distance along the geostrophic flow, $y$ is in the horizontal direction perpendicular to the geostrophic flow and $z$ is in the vertical direction as shown in Fig. 1. $\bar{u}$ and $\bar{v}$ are the $x$ and $y$ components of the Ekman flow velocity, respectively.

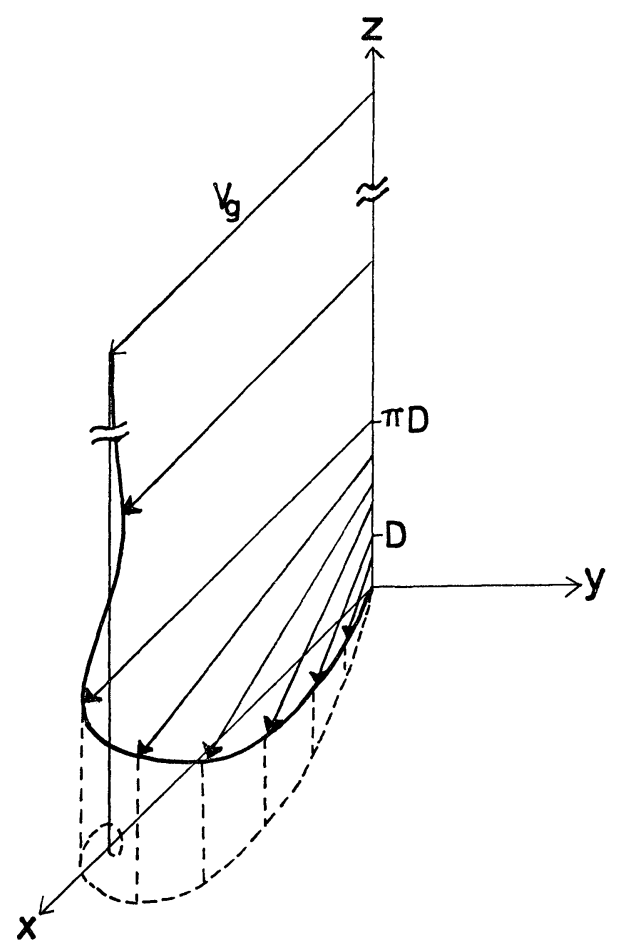

Fig. 1. A diagram of the Ekman boundary layer flow and the coordinate system.
The pertinent equations for small-amplitude perturbations superposed on the Ekman flow (2.1) are written as

$$
\begin{aligned}
& \frac{\partial \boldsymbol{v}^{\prime}}{\partial t}+\ddot{\boldsymbol{v}} \cdot \nabla \boldsymbol{v}^{\prime}+w^{\prime} \frac{\partial \overline{\boldsymbol{v}}}{\partial z}+f \boldsymbol{k} \times \boldsymbol{v}^{\prime} \\
& =-C_{p} \theta_{0} \nabla \pi^{\prime}+\frac{\theta^{\prime}}{\theta_{0}} g \boldsymbol{k}+\nu \nabla^{2} \boldsymbol{v}^{\prime}, \\
& \frac{\partial \theta^{\prime}}{\partial t}+\ddot{\boldsymbol{v}} \cdot \nabla^{\theta^{\prime}+w^{\prime}} \frac{\partial \bar{\theta}}{\partial z}=\kappa \nabla^{2} \theta^{\prime}, \\
& \nabla \cdot \boldsymbol{v}^{\prime}=0,
\end{aligned}
$$

where $\overline{\boldsymbol{v}}=\bar{u} \boldsymbol{i}+\bar{v} \boldsymbol{j}, \quad \boldsymbol{v}^{\prime}=u^{\prime} \boldsymbol{i}+v^{\prime} \boldsymbol{j}+w^{\prime} \boldsymbol{k}$,

$$
\nabla=\boldsymbol{i} \frac{\partial}{\partial x}+\boldsymbol{j} \frac{\partial}{\partial y}+\boldsymbol{k} \frac{\partial}{\partial z},
$$

$\boldsymbol{i}, \boldsymbol{j}$ and $\boldsymbol{k}$ are the unit vectors in the $x, y$ and $z$ directions, respectively, $\pi=\left(p / p_{0}\right)^{(\gamma-1) / \gamma}, p$ is the pressure, $p_{0}$ is the reference pressure, $\gamma=C_{p} / C_{v}$, $C_{p}$ is the specific heat at the constant pressure, $C_{v}$ is the specific heat at the constant volume, $\theta$ is the potential temperature, $g$ is the acceleration due to gravity and $\kappa$ is the coefficient of thermometric conductivity. Here the potential temperature in the basic flow is expressed by

$$
\bar{\theta}=\theta_{0}-\beta\left(z-\frac{H}{2}\right),
$$

where $H$ is the thickness of the fluid layer and $\beta$ denotes the vertical gradient of potential temperature assumed as constant.

The boundary conditions at the lower and upper surfaces adopted here are

$$
\left.\begin{array}{l}
u^{\prime}=v^{\prime}=w^{\prime}=\theta^{\prime}=0 \quad \text { at } \quad z=0, \\
\frac{\partial u^{\prime}}{\partial z}=\frac{\partial v^{\prime}}{\partial z}=w^{\prime}=\theta^{\prime}=0 \text { at } \quad z=H,
\end{array}\right\}
$$

where $H$ is taken to be high enough.

\section{Dimensionless forms}

Now it is convenient to transform the equations (2.1), (2.6), (2.3), (2.4) and (2.5) into the dimensionless forms, i.e.,

$$
\left.\begin{array}{l}
\bar{u}^{*}=1-e^{-z^{*}} \cos z^{*} \\
\bar{v}^{*}=e^{-z *} \sin z^{*},
\end{array}\right\}
$$




$$
\begin{aligned}
& \bar{\theta}^{*}=\frac{\theta_{0}}{\beta D}-\left(z^{*}-\frac{h}{2}\right), \\
& R_{e}\left(\frac{\partial \boldsymbol{v}^{\prime *}}{\partial t^{*}}+\overline{\boldsymbol{v}}^{*} \nabla^{*} \boldsymbol{v}^{\prime *}+w^{\prime *} \frac{\partial \overline{\boldsymbol{v}}^{*}}{\partial z^{*}}\right)+2 \boldsymbol{k} \times \boldsymbol{v}^{\prime *} \\
& \quad=-R_{e} \nabla^{*} \pi^{\prime *}+R_{e} R_{i} \theta^{\prime *} \boldsymbol{k}+\nabla^{* 2} \boldsymbol{v}^{\prime *} \\
& P_{r} R_{e}\left(\frac{\partial \theta^{\prime *}}{\partial t^{*}}+\overline{\boldsymbol{v}}^{*} \cdot \nabla^{*} \theta^{\prime *}+w^{\prime *} \frac{\partial \bar{\theta}^{*}}{\partial z^{*}}\right)=\nabla^{* 2 \theta^{\prime *}} \\
& \nabla^{*} \cdot \boldsymbol{v}^{\prime *}=0 .
\end{aligned}
$$

(3.3) where

The asterisks signify the respective dimensionless quantities. Here we introduced the following characteristic values to nondimensionalize the equations:

$$
\begin{aligned}
\left(x^{*}, y^{*}, z^{*}\right) & =(x, y, z) / D, \\
\left(\overline{\boldsymbol{v}}^{*}, \boldsymbol{v}^{*}\right) & =\left(\overline{\boldsymbol{v}}, \boldsymbol{v}^{\prime}\right) / V_{g}, \\
\left(\bar{\theta}^{*}, \theta^{*}\right) & =\left(\bar{\theta}, \theta^{\prime}\right) /(\beta D), \\
t^{*} & =t^{\prime} V_{g} / D, \\
\pi^{*} & =\pi^{\prime} C_{p} \theta_{0} / V_{g}^{2} .
\end{aligned}
$$

The Reynolds number $R_{e}$, the Richardson number $R_{i}$ and the Prandtl number $P_{r}$ are defined, respectively as follows:

$$
R_{e}=\frac{V_{g} D}{\nu}, R_{i}=\frac{g \beta D^{2}}{\theta_{0} V_{g}^{2}}, \quad P_{r}=\frac{\nu}{\kappa} .
$$

Hence the Rayleigh number $R_{a}$ may be expressed by

$$
R_{a}=\frac{g \beta H^{4}}{\theta_{0} \kappa \nu}=P_{r} R_{e}^{2} R_{i} h^{4},
$$

where $h=H / D$. In the following the asterisk notation " $*$ " will be neglected for simplification.

Eliminating $u^{\prime}, v^{\prime}$ and $\pi^{\prime}$ from (3.3), (3.4) and (3.5), we obtain the following equations.

$$
\begin{aligned}
& {\left[\nabla^{2}-R_{e}\left(\frac{\partial}{\partial t}+\bar{u} \frac{\partial}{\partial x}+\bar{v} \frac{\partial}{\partial y}\right)\right] \zeta^{\prime}} \\
& +\left[R_{e}\left(\frac{\partial \bar{u}}{\partial z} \frac{\partial}{\partial y}-\frac{\partial \bar{v}}{\partial z} \frac{\partial}{\partial x}\right)+2 \frac{\partial}{\partial z}\right] w^{\prime}=0 \\
& {\left[\nabla^{4}-R_{e}\left(\frac{\partial}{\partial t}+\bar{u} \frac{\partial}{\partial x}+\bar{v} \frac{\partial}{\partial y}\right) \nabla^{2}\right.} \\
& \left.+R_{e}\left(\frac{\partial^{2} \bar{u}}{\partial z^{2}} \frac{\partial}{\partial x}+\frac{\partial^{2} \bar{v}}{\partial z^{2}} \frac{\partial}{\partial y}\right)\right] w^{\prime} \\
& \quad-2 \frac{\partial \zeta^{\prime}}{\partial z}+R_{e} R_{i} \nabla_{H}^{2} \theta^{\prime}=0
\end{aligned}
$$

$$
\begin{gathered}
{\left[\nabla^{2}-P_{r} R_{e}\left(\frac{\partial}{\partial t}+\bar{u} \frac{\partial}{\partial x}+\bar{v} \frac{\partial}{\partial y}\right)\right] \theta^{\prime}} \\
-P_{r} R_{e} \frac{\partial \bar{\theta}}{\partial z} w^{\prime}=0,
\end{gathered}
$$

$$
\nabla_{H}^{2}=\frac{\partial^{2}}{\partial x^{2}}+\frac{\partial^{2}}{\partial y^{2}} \text { and } \zeta^{\prime}=\frac{\partial v^{\prime}}{\partial x}-\frac{\partial u^{\prime}}{\partial y} .
$$

Furthermore a perturbation is assumed of the form, i.e.,

$$
\left(\begin{array}{c}
\zeta^{\prime}(x, y, z, t) \\
w^{\prime}(x, y, z, t) \\
\theta^{\prime}(x, y, z, t)
\end{array}\right)=\left(\begin{array}{r}
Z(z) \\
W(z) \\
\Theta(z)
\end{array}\right) \exp \left\{i\left(k_{x} x+k_{y} y\right)+\sigma t\right\}
$$

where $k_{x}$ and $k_{y}$ are the wavenumbers in the $x$ and $y$ directions, respectively, $\sigma$ is the complex frequency, i.e., $\sigma=\sigma_{r}+i \sigma_{i}$. Substitution of (3.13) into (3.9) (3.11) leads to the following ordinary differential equations:

$$
\begin{aligned}
& {\left[\frac{d^{2}}{d z^{2}}-k^{2}-R_{e}\left(\sigma+i k_{x} \bar{u}+i k_{y} \bar{v}\right)\right] Z} \\
& +\left[i R_{e}\left(k_{y} \frac{d \bar{u}}{d z}-k_{x} \frac{d \bar{v}}{d z}\right)+2 \frac{d}{d z}\right] W=0 \\
& \left\{\frac{d^{4}}{d z^{4}}-\left[2 k^{2}+R_{e}\left(\sigma+i k_{x} \bar{u}+i k_{y} \bar{v}\right)\right] \frac{d^{2}}{d z^{2}}\right. \\
& +k^{4}+k^{2} R_{e}\left(\sigma+i k_{x} \bar{u}+i k_{y} \bar{v}\right) \\
& \left.+i R_{e}\left(k_{x} \frac{d^{2} \bar{u}}{d z^{2}}+k_{y} \frac{d^{2} \bar{v}}{d z^{2}}\right)\right\} W \\
& -2 \frac{d Z}{d z}-k^{2} R_{e} R_{i} \Theta=0
\end{aligned}
$$$$
\left[\frac{d^{2}}{d z^{2}}-k^{2}-P_{r} R_{\varepsilon}\left(\sigma+i k_{x} \bar{u}+i k_{y} \bar{v}\right)\right] \Theta
$$$$
-P_{r} R_{e} \frac{d \bar{\theta}}{d z} W=0,
$$

where $k^{2}=k_{x}^{2}+k_{y}^{2}$.

The boundary conditions at the upper and lower boundaries are

$$
\begin{aligned}
& Z=W=\frac{d W}{d z}=\Theta=0 \quad \text { at } \quad z=0, \\
& \frac{d Z}{d z}=W=\frac{d^{2} W}{d z^{2}}=\Theta=0 \text { at } z=h .
\end{aligned}
$$

The equations (3.14) (3.16) with the boundary conditions (3.17) constitute an eigenvalue problem 
in which the frequency $\sigma$ is the eigenvalue, $Z, W$ and $\Theta$ are the eigenfunctions and $k_{x}, k_{y}, R_{e}, R_{i}$, $P_{r}$ and $h$ are the specified parameters. These equations will be solved numerically in the manner described in Section 5.

\section{Kinetic energy equation}

Making use of the equation of motion (3.3) and the equation of mass conservation (3.5) with the boundary conditions (2.7), we may obtain the equation of perturbation kinetic energy as follows:

$$
\frac{\partial}{\partial t}<K^{\prime}>=<\bar{K}, K^{\prime}>+<P, K^{\prime}>-<K^{\prime} D>,
$$

where

$$
\begin{aligned}
&\left.<K^{\prime}\right\rangle=\frac{1}{2}\left\langle\boldsymbol{v}^{\prime 2}\right\rangle=\frac{1}{2}\left\langle u^{\prime 2}+v^{\prime 2}+w^{\prime 2}\right\rangle, \\
&\left.<\bar{K}, K^{\prime}\right\rangle=-\left\langle u^{\prime} w^{\prime} \frac{d \bar{u}}{d z}\right\rangle-\left\langle v^{\prime} w^{\prime} \frac{d \bar{v}}{d z}\right\rangle, \\
&=-\left\langle v^{\prime} w^{\prime} \frac{d \bar{v}_{\|}}{d z}\right\rangle-\left\langle v_{\perp}{ }^{\prime} w^{\prime} \frac{d \bar{v}_{\perp}}{d z}\right\rangle, \\
&\left.<P, K^{\prime}\right\rangle=R_{i}\left\langle\theta^{\prime} w^{\prime}\right\rangle, \\
&\left.<K^{\prime} D\right\rangle=-R_{e}^{-1}\left\langle\boldsymbol{v}^{\prime} \cdot \nabla^{2} \boldsymbol{v}^{\prime}\right\rangle \\
&=-R_{e}^{-1}\left\langle u^{\prime} \nabla^{2} u^{\prime}+v^{\prime} \nabla^{2} v^{\prime}+w^{\prime} \nabla^{2} w^{\prime}\right\rangle,
\end{aligned}
$$

and angular brackets denote an average over one wavelength in the $x$ and $y$ directions and an entire depth of the layer concerned. Parallel and normal components of the basic flow and of the perturbation horizontal velocity to a roll axis of the perturbation are denoted by $\bar{v}_{\|}, \bar{v}_{\perp}, v_{\|}{ }^{\prime}$ and $v_{\perp}{ }^{\prime}$, respectively. The first term on the right-hand side of (4.1) represents conversion of energy from the basic flow into the perturbation through the action of the Reynolds stress. The second term represents conversion from the potential energy to the perturbation kinetic energy through the upward heat transport. The last term is the rate at which the perturbation kinetic energy is dissipated by viscous processes.

\section{Computational procedure}

A common feature of a boundary layer flow is that significant shear of the flow is confined to the vicinity of the boundary surface. Asai and Nakasuji (1971) examined feasibility of applying a centered finite-difference method with non-uniform grid distance to stability analysis of boundary layer flows. A method similar to Asai and Nakasujis' is adopted in the following.

We introduce a new vertical coordinate, $z_{*}$, defined as

$$
z_{*}=\frac{1-e^{-a z}}{1-e^{-a h}},
$$

where $a$ is a constant parameter. By making use of the new vertical coordinate $z_{*}$, we may rewrite (3.14) (3.17) as follows:

$$
\begin{aligned}
& {\left[a^{2}\left(z_{*}-b\right)^{2} \frac{d^{2}}{d z_{*}{ }^{2}}+a^{2}\left(z_{*}-b\right) \frac{d}{d z_{*}}-k^{2}\right.} \\
& \left.-R_{e}\left(\sigma+i k_{x} \bar{u}+i k_{y} \bar{v}\right)\right] Z+\left[i R _ { e } \left(k_{y} \frac{d \bar{u}}{d z}\right.\right. \\
& \left.\left.-k_{x} \frac{d \bar{v}}{d z}\right)-2 a\left(z_{*}-b\right) \frac{d}{d z_{*}}\right] W=0, \\
& \left\{a^{4}\left(z_{*}-b\right)^{4} \frac{d^{4}}{d z_{*^{4}}^{4}}+6 a^{4}\left(z_{*}-b\right)^{3} \frac{d^{3}}{d z_{*}^{3}}\right. \\
& +a^{2}\left(z_{*}-b\right)^{2}\left(7 a^{2}-2 k^{2}\right) \frac{d^{2}}{d z_{*}{ }^{2}} \\
& +a^{2}\left(z_{*}-b\right)\left(a^{2}-2 k^{2}\right) \frac{d}{d z_{*}} \\
& -a^{2} R_{e}\left(\sigma+i k_{x} \bar{u}+i k_{y} \bar{v}\right)\left[\left(z_{*}-b\right)^{2} \frac{d^{2}}{d z_{*}{ }^{2}}\right. \\
& \left.+\left(z_{*}-b\right) \frac{d}{d z_{*}}\right]+k^{4}+k^{2} R_{e}\left(\sigma+i k_{x} \bar{u}+i k_{y} \bar{v}\right) \\
& \left.+i R_{e}\left(k_{x} \frac{d^{2} \bar{u}}{d z^{2}}+k_{y} \frac{d^{2} \bar{v}}{d z^{2}}\right)\right\} W \\
& +2 a\left(z_{*}-b\right) \frac{d Z}{d z_{*}}-k^{2} R_{e} R_{i} \Theta=0, \\
& {\left[a^{2}\left(z_{*}-b\right)^{2} \frac{d}{d z_{*}{ }^{2}}+a^{2}\left(z_{*}-b\right) \frac{d}{d z_{*}}\right.} \\
& \left.-k^{2}-P_{r} R_{e}\left(\sigma+i k_{x} \bar{u}+i k_{y} \bar{v}\right)\right] \Theta \\
& -P_{r} R_{e} \frac{d \bar{\theta}}{d z} W=0
\end{aligned}
$$

$$
\begin{aligned}
& Z=W=\frac{d W}{d z_{*}}=\Theta=0 \text { at } z_{*}=0, \\
& \begin{aligned}
\frac{d Z}{d z_{*}} & =W=\left(z_{*}-b\right) \frac{d^{2} W}{d z_{*}{ }^{2}}+\frac{d W}{d z_{*}} \\
& =\Theta=0 \quad \text { at } \quad z_{*}=1,
\end{aligned}
\end{aligned}
$$




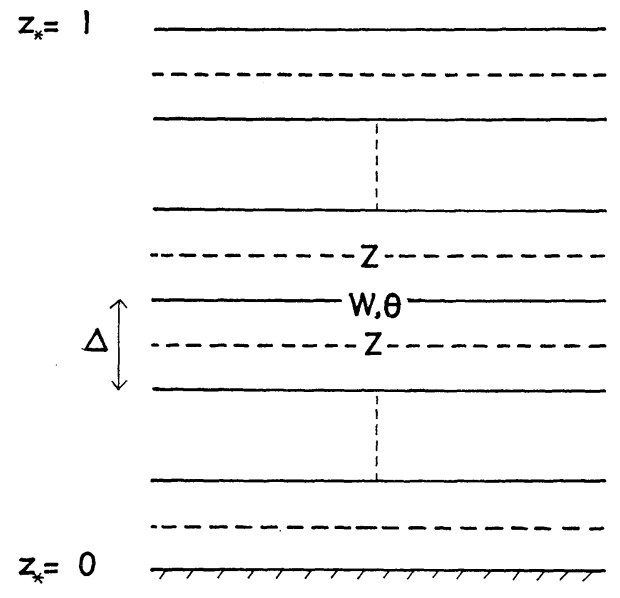

Fig. 2. Multi-layer representation of dependent variables.

where $b=1 /\left(1-e^{-a h}\right)$. Note that the derivatives of $\bar{u}, \bar{v}$ and $\bar{\theta}$ are expressed with respect to $z$ for convenience. Since a uniform grid spacing in the $z_{*}$ coordinate is used, (5.1) indicates that a greater positive value of $a$ specifies the grid spacing so as to achieve higher resolution in the lower levels near the boundary and lower resolution far from the boundary.

Approximation of $(5.2) \sim(5.5)$ by the centered finite-difference forms at the levels shown in Fig. 2 yields

$$
\begin{aligned}
a^{2}\left(z_{*}\right. & -b) \delta^{2} Z+a^{2}\left(z_{*}-b\right) \delta \hat{Z} \\
& -\left[k^{2}+R_{e}\left(\sigma+i k_{x} \bar{u}+i k_{y} \bar{v}\right)\right] Z \\
& +i R_{e}\left(k_{y} \frac{d \bar{u}}{d z}-k_{x} \frac{d \bar{v}}{d z}\right)\left(\hat{W}-\frac{\Delta^{2}}{8} \delta^{2} \hat{W}\right) \\
& -2 a\left(z_{*}-b\right) \delta W=0, \\
a^{4}\left(z_{*}-b\right)^{4} \delta^{4} W+6 a^{4}\left(z_{*}-b\right)^{3} \delta^{3} \hat{W} & +a^{2}\left(z_{*}-b\right)^{2}\left(7 a^{2}-2 k^{2}\right) \delta^{2} W \\
& +a^{2}\left(z_{*}-b\right)\left(a^{2}-2 k^{2}\right) \delta \hat{W}-a^{2} R_{e}(\sigma \\
& \left.+i k_{x} \bar{u}+i k_{y} \bar{v}\right)\left[\left(z_{*}-b\right)^{2}\left(\delta^{2} W-\frac{\Delta^{2}}{12} \delta^{4} W\right)\right. \\
& \left.+\left(z_{*}-b\right)\left(\delta \hat{W}-\frac{\Delta^{2}}{6} \delta^{3} \hat{W}\right)\right] \\
& +\left[k^{4}+k^{2} R_{e}\left(\sigma+i k_{x} \bar{u}+i k_{y} \bar{v}\right)\right. \\
& \left.+i R_{e}\left(k_{x} \frac{d^{2} \bar{u}}{d z^{2}}+k_{y} \frac{d^{2} \bar{v}}{d z^{2}}\right)\right] W \\
+ & 2 a\left(z_{*}-b\right) \delta Z-k^{2} R_{e} R_{i} \Theta=0,
\end{aligned}
$$

$$
\begin{aligned}
& a^{2}\left(z_{*}-b\right)^{2} \delta^{2} \Theta+a^{2}\left(z_{*}-b\right) \delta \hat{\Theta} \\
& \quad-\left[k^{2}+P_{r} R_{e}\left(\sigma+i k_{x} \bar{u}+i k_{y} \bar{v}\right)\right] \Theta \\
& \quad-P_{r} R_{e} \frac{d \bar{\theta}}{d z} W=0, \\
& Z=W=\delta \hat{W}=\Theta=0 \quad \text { at } \quad z_{*}=0, \\
& \delta Z=W=\left(z_{*}-b\right) \delta^{2} W+\delta \hat{W}=\Theta=0 \\
& \quad \text { at } \quad z_{*}=1,
\end{aligned}
$$

where

$$
\left.\begin{array}{l}
\delta Z=\frac{1}{\Delta}\left[Z\left(z_{*}+\frac{\Delta}{2}\right)-Z\left(z_{*}-\frac{\Delta}{2}\right)\right], \\
\hat{Z}=\frac{1}{2}\left[Z\left(z_{*}+\frac{\Delta}{2}\right)+Z\left(z_{*}-\frac{\Delta}{2}\right)\right], \\
\delta^{2} Z=\delta(\delta Z), \text { etc. }
\end{array}\right\}
$$

A grid distance is denoted by $\Delta$ which is equal to $1 / n$ for an $n$-layer model. The finite-difference approximation by Lilly (1966) is used in deriving (5.6) (5.8). Only a difference of Lilly's method from the centered differencing customarily used is that $\Delta^{2}$-order terms are included in the terms associated with $R_{e}$.

Hence, (5.6) (5.9) are a set of linear algebraic equations in unknown variables $W$, (A) and $Z$ at the respective levels and may be written symbolically in the following matrix:

$$
(\boldsymbol{A}-\boldsymbol{B} \sigma) \boldsymbol{X}=0,
$$

where $\boldsymbol{A}$ and $\boldsymbol{B}$ are matrices and $\boldsymbol{X}$ is the vector of the eigenfunction solution for $W, \Theta$ and $Z$. Multiplying (5.11) by the inverse of $\boldsymbol{B}$, we obtain

$$
\left(\boldsymbol{B}^{-1} \boldsymbol{A}-\sigma \boldsymbol{I}\right) \boldsymbol{X}=0 .
$$

where $\boldsymbol{I}$ is the unit matrix and $\boldsymbol{B}^{-1}$ is the inverse matrix of $\boldsymbol{B}$. Provided that $\boldsymbol{X}$ is to be non-zero solution, the determinant of matrix $\left(\boldsymbol{B}^{-1} \boldsymbol{A}-\sigma \boldsymbol{I}\right)$ must vanish, i.e.,

$$
\left|\boldsymbol{B}^{-1} \boldsymbol{A}-\boldsymbol{\sigma} \boldsymbol{I}\right|=0 .
$$

Thus eigenvalue $\sigma$ and the eigenvector $\boldsymbol{X}$ can be calculated for given parameters.

With the aid of (3.5) and (3.12) amplitude functions $U$ and $V$ for $u^{\prime}$ and $v^{\prime}$ respectively, if necessary, can be calculated by the following equations:

$$
\begin{aligned}
& U=\frac{i}{k^{2}}\left(k_{y} Z+k_{x} \delta W\right), \\
& V=\frac{i}{k^{2}}\left(-k_{x} Z+k_{y} \delta W\right) .
\end{aligned}
$$




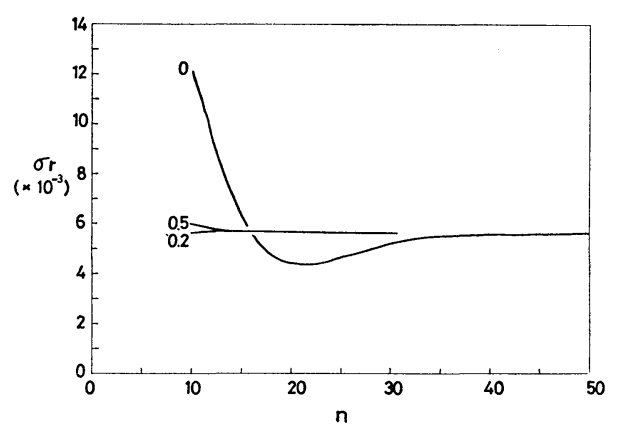

Fig. 3. The calculated amplification rates of an unstable perturbation versus the number of the layer subdivision $n$ for different values of $a$. $R_{e}=110, R_{i}=0, k=0.3, \varepsilon=-10^{\circ}$ and $h=10.8$.

Furthermore we note that amplitude functions $V_{\|}$and $V_{\perp}$ for $v_{\| \prime}{ }^{\prime}$ and $v_{\perp}{ }^{\prime}$ are given respectively by

$$
\begin{aligned}
& V_{\text {!! }}=\frac{i}{k} Z, \\
& V_{\perp}=\frac{i}{k} \delta W .
\end{aligned}
$$

A series of calculations are carried out for given parameters such as $R_{e}, R_{i}, P_{r}, k_{x}, k_{y}$ and $h$.

An efficiency of the non-uniform grid system used here was examined in detail. One of the results obtained is shown in Fig. 3 which depicts variations of calculated values of amplification rate of an unstable perturbation with the number of subdivision $n$ for different values of $a$. It is apparent that the calculated amplification rates approach a particular value to be regarded as the exact solution when $n$ is greater. As is shown in Fig. 3, a 10-layer subdivision may provide a solution accurate enough to retain an error within $10 \%$ for $a=0.2$ and 0.5 , while for the uniform grid spacing, 30 or more layers subdivision is required. Most of the calculations in this paper were made by using the 10-layer subdivision in the non-uniform grid system. However, the uniform grid system was used for the cases in which unstable perturbations of the type III to be described below were dominant.

An optimal value of $a$ for the most efficient calculation exists between 0.1 and 1.0 and increases with increasing values of $k$ and $\varepsilon$, where $\varepsilon \equiv-\tan ^{-1}\left(\frac{k_{x}}{k_{y}}\right)$ represents the orientation angle of a roll perturbation. Note that a coefficient of $W$ in the finite-difference form of (5.9) may vanish for a certain value of $a$, for instance $a=0.3$ when $h=10$ and $n=10$. Such a value of $a$ and its neighboring values should not be used.

\section{Stability properties}

Theoretical studies of stability of the Ekman flow in a homogeneous fluid made by Lilly (1966), Faller and Kaylor (1966), Brown (1970) and others confirmed the presence of two distinct types of unstable waves. One type of unstable waves appears at $R_{e} \simeq 55$ and the other type of unstable waves develops at values of $R_{e}$ larger than 150. Let us now examine possible effects of thermally unstable stratification on the stability properties of these two types by varying values of $R_{a}$ for the cases of $R_{e}=100$ and $R_{e}=1,000$ for which either of the instabilities may be favored. Fig. 4 (a), (b), (c) and (d) show amplification rates as functions of wavenumber $k$ and orientation angle $\varepsilon$ of a roll perturbation for (a) $R_{e}=$ $10^{2}, R_{a}=10^{4}$; (b) $R_{e}=10^{2}, R_{a}=10^{5}$; (c) $R_{e}=10^{3}$, $R_{a}=10^{6}$; (d) $R_{e}=10^{3}, R_{a}=10^{7}$, respectively. $P_{r}=$ 1 and $h=10$ are assumed unless specifically stated otherwise. Amplification rates denoted by solid and dashed lines are in units of $V_{g}\left(\frac{f}{2 \nu}\right)^{1 / 2}$, wavenumber of the radial coordinate is in units of $\left(\frac{f}{2 \nu}\right)^{1 / 2}$ and the angular coordinate is the orientation angle which is defined as $-\tan ^{-1}$ $\left(\frac{k_{x}}{k_{y}}\right)$ and is measured counterclockwise from the direction of the geostrophic flow to the axis of the perturbation roll. Figures 4 (a) and (c) exhibit a shift of a preferred mode of unstable perturbations, of which amplification rate attains a maximum, from an angle of negative orientation to a positive one. The former unstable perturbation at lower Reynolds number is designated by type I and the latter at higher Reynolds number by type II just for convenience. Comparisons between Figs. 4 (a) and (b) and between (c) and (d) suggest that there may appear another type of instability having no specific orientation angle of a preferred unstable perturbation as Rayleigh number increases. Variation of the amplification rate of unstable perturbations with $R_{a}$ and $k$ are shown in further detail in Fig. 5 for $R_{e}=10^{3}$ and $\varepsilon=20^{\circ}$. As is shown in Fig. 5, the most unstable wavenumber for a given Rayleigh number 


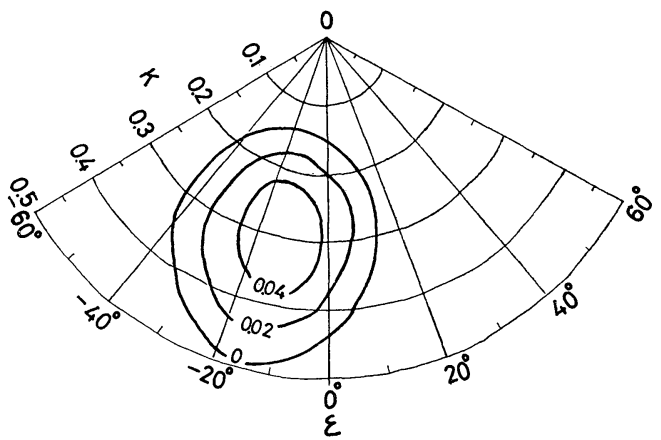

(a)

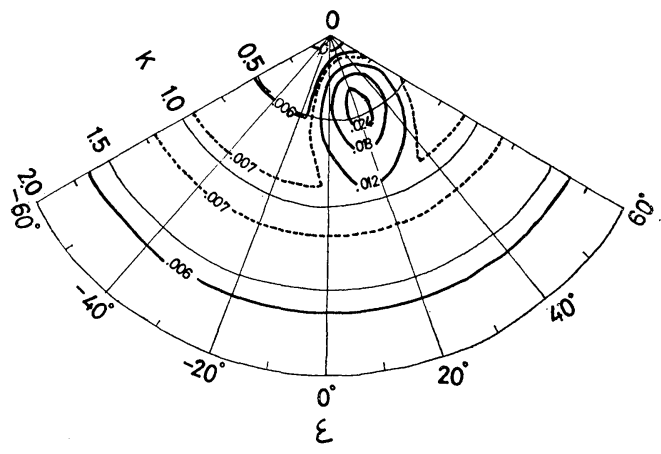

(c)

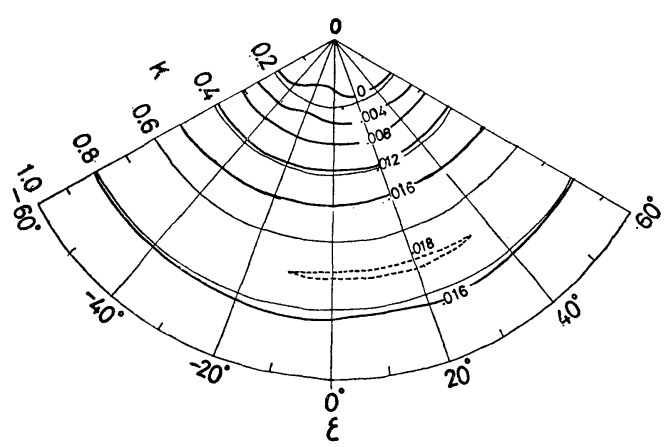

(b)

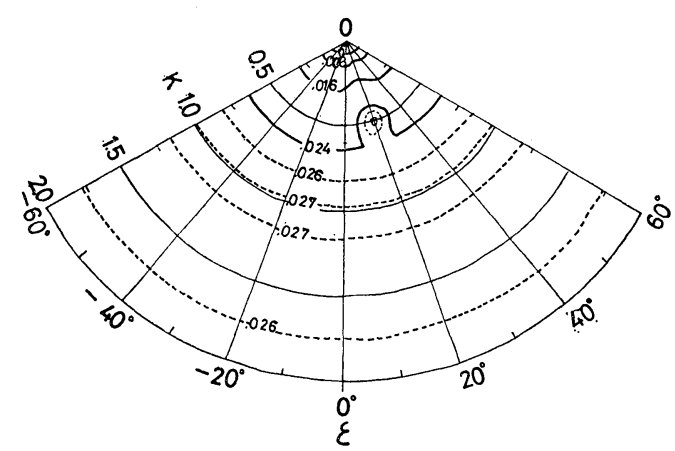

(d)

Fig. 4. The amplification rates of unstable perturbations as functions of wavenumber, $k$, and orientation angle, $\varepsilon$ : (a) $R_{e}=10^{2}$ and $R_{a}=10^{4}$; (b) $R_{e}=10^{2}$ and $R_{a}=10^{5}$; (c) $R_{e}=10^{3}$ and $R_{a}=10^{6}$; (d) $R_{e}=10^{3}$ and $R_{a}=10^{7}$.

denoted by a dash-dotted line shifts from 0.5 to 1 abruptly at $R_{a} \simeq 10^{7}$, and the amplification rate increases rapidly with increasing value of $R_{a}$ larger than $10^{7}$ while slight change of the amplification rate with Rayleigh number is observed for $R_{a}<10^{7}$.

Variations of the amplification rates, $\sigma_{r} R_{e}$, nondimensionalized in terms of $f / 2$ with Reynolds number for different values of $\varepsilon\left(-10^{\circ}, 20^{\circ}, 90^{\circ}\right)$ in the case of $R_{a}=2 \times 10^{4}$ and $k=0.5$ are shown in Fig. 6 to examine an influence of the vertical shear on the amplification rate. The instability domain is divided into two portions: one is a region of $R_{e}>100$ in which the amplification rate increases with increasing $R_{e}$, while the other is a region of $R_{e}<50$ in which the amplification rate decreases with increasing $R_{e}$ and depends slightly on the orientation angle. It seems that the former is of either the type I or the type II and the latter is of thermal instability which is hereafter referred to as type III.

The amplification rates of preferred perturba-

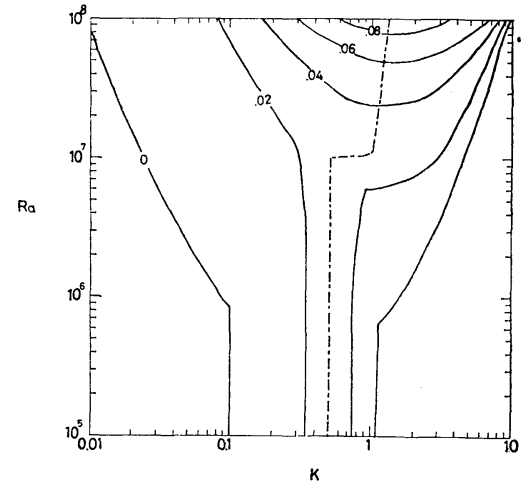

Fig. 5. The amplification rates as a function of Rayleigh number, $R_{a}$, and wavenumber, $k$, for the case of $R_{e}=10^{3}$ and $\varepsilon=20^{\circ}$. A dash-dotted line indicates a preferred wavenumber, for a given value of $R_{a}$. 


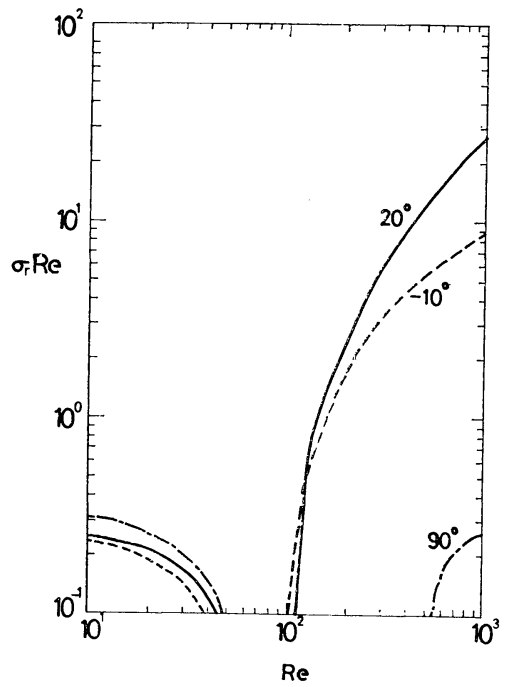

Fig. 6. The amplification rates, $\sigma_{r} R_{e}$, versus the Reynolds number $R_{e}$ for different values of orientation angle $\varepsilon . R_{a}=2 \times 10^{4}$ and $k=0.5$. Solid lines correspond to $\varepsilon=20^{\circ}$, dashed lines to $\varepsilon=-10^{\circ}$ and dashdotted lines to $\varepsilon=90^{\circ}$.

tions, $\sigma_{r} R_{e}$, are shown as a function of $R_{a}$ and $R_{e}$ in Fig. 7. The value of $\sigma_{r} R_{e}$ in Fig. 7 is the maximum amplification rate among unstable perturbations of different values of $k$ and $\varepsilon$ for given values of $R_{e}$ and $R_{a}$. The corresponding Richardson numbers are denoted by dashed lines. An inspection of Fig. 7. indicates that the unstable domain may be divided into two regions by a dotted line located along the dashed line of $R_{i}=10^{-3}$ : one is a region of $R_{i} \gtrsim 10^{-3}$ in which the amplification rate increases with increasing $R_{a}$ and changes slightly with $R_{\ell}$, while the other is a region of $R_{i} \lesssim 10^{-3}$ in which the amplification rate increases with increasing $R_{e}$ and shows slight change with $R_{a}$. Note that the lowest Rayleigh number at which instability sets in for a small value of $R_{e}$ is about $1.5 \times 10^{4}$ which agrees with the critical Rayleigh number for thermal instability in a rotating fluid obtained by Chandrasekhar and Elbert (1955) and the lowest Reynolds number at which instability sets in for a small value of $R_{a}$ is coincident with the critical Reynolds number 55, for instability of the neutral Ekman layer flow given by Lilly (1966).

It is thus suggested from Figs. 4, 5, 6 and 7

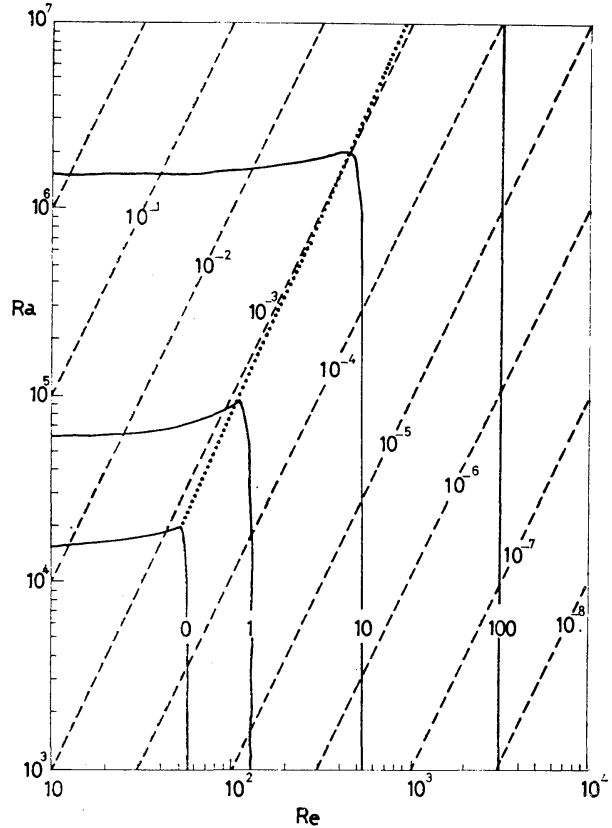

Fig. 7. The amplification rates, $\sigma_{r} R_{e}$, of the respective preferred perturbations as a function of the Rayleigh number $R_{a}$ and the Reynolds number $R_{e}$. Dashed lines indicate the Richardson number, $R_{i}$.

that there may appear three different types of unstable perturbations. Unstable perturbations of the type I are dominant in a range of $55<R_{e}$ $<150$ and $R_{i}<10^{-3}$, and their amplification rates increase rapidly with increasing $R_{e}$ but are slightly dependent on $R_{a}$. The orientation angle of a preferred perturbation is about $-10^{\circ}$ with respect to the geostrophic flow and the wavenumber is 0.3 which means the wavelength of $21 \mathrm{D}$. Unstable perturbations of the type II prevail when $R_{e}>150$ and $R_{i}<10^{-3}$ and their amplification rates increase with increasing $R_{e}$ but are slightly dependent on $R_{a}$. A preferred perturbation of this type has the orientation angle of $20^{\circ}$ and the wavenumber of 0.5 which corresponds to the wavelength of $12 \mathrm{D}$. The characteristics of instability of these two types are in good agreement respectively those shown by Lilly (1966) for the neutral Ekman layer flow. The amplification rates of unstable perturbations of the type III increase as $R_{a}$ increases but slightly depend on $R_{e}$. A preferred perturbation has the wavenumber of about unity and no 
definite angle of orientation.

Fig. 8 shows the phase velocities $c\left(=-\sigma_{i} / k\right)$ in units of $V_{g}$ of unstable perturbations of the

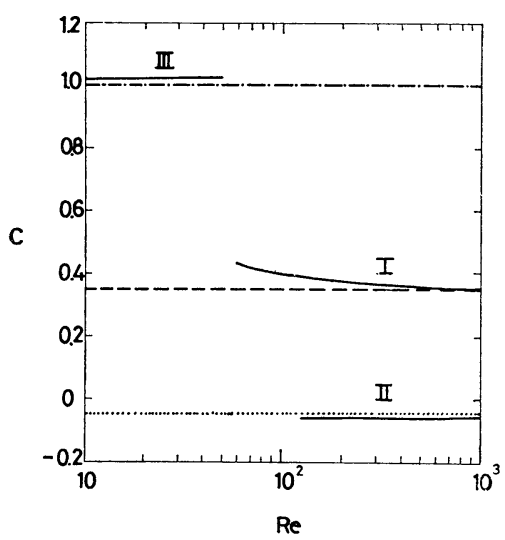

Fig. 8. The phase velocities of three unstable perturbations of the types I, II and III, which are of (I) $\varepsilon=-10^{\circ}$, (II) $\varepsilon=$ $20^{\circ}$ and (III) $\varepsilon=90^{\circ}$, as functions of $R_{e}$, respectively. $k=$ 0.5 and $R_{a}=2 \times 10^{4}$. The dash-dotted line, the dashed line and the dotted line indicate the velocity components of the geostrophic flow and of the Ekman flow at the lowest inflection points perpendicular to the axis of perturbation rolls, respectively. three different types as a function of $R_{e}$. They are the same examples as in Fig. 6 but $\varepsilon=-10^{\circ}$. for the type I, $\varepsilon=20^{\circ}$ for the type II and $\varepsilon=90^{\circ}$ for the type III. The unstable perturbation of the type III seems to move at the velocity of the geostrophic flow denoted by the dash-dotted line, while those of the types I and II move at the velocities around the lowest inflection points in the vertical profiles of the velocity components of the Ekman flow perpendicular to the roll axes denoted by the dashed line and the dotted line, respectively.

\section{Structures of unstable perturbations and the associated energy conversion}

In this section we make an attempt to clarify characteristics of the three types of instabilities separated from one another in the previous section. Figs. 9, 10 and 11 depict vertical structures of unstable perturbations of the types I, II and III, respectively, for which the three particular sets of parameters are taken as follows:

(1) $k=0.3, \varepsilon=-10^{\circ}, R_{e}=10^{2}$, $R_{i}=10^{-4}$ for the type I,

(2) $k=0.5, \varepsilon=20^{\circ}, R_{e}=10^{3}$, $R_{i}=10^{-4}$ for the type II and

(3) $k=1, \varepsilon=0^{\circ}, R_{e}=10^{2}$, $R_{i}=10^{-2}$ for the type III.

Vertical profiles of the amplitudes and the phase angles of $w^{\prime}, \theta^{\prime}, v_{\|}{ }^{\prime}$ and $v_{\perp}{ }^{\prime}$ are illustrated by the solid lines, the dashed lines, the dotted lines and the dash-dotted lines, respectively. Solid

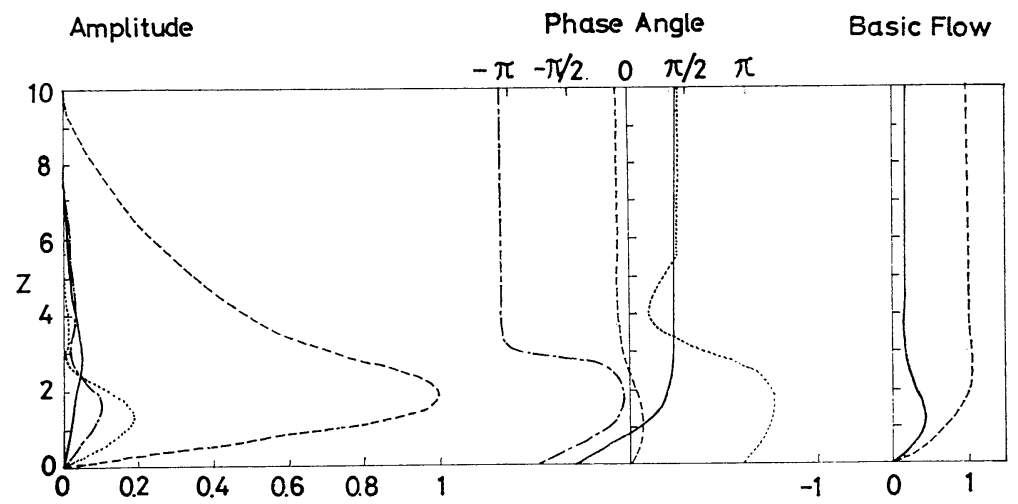

Fig. 9. Vertical structure of an unstable perturbation of the type I. Amplitudes and phase angles of $w^{\prime}, \theta^{\prime}, v_{11}{ }^{\prime}$ and $v_{\perp}{ }^{\prime}$ are denoted by solid, dashed, dotted and dash-dotted lines, respectively. Vertical profiles of $\overline{v_{\|}}$and $\overline{v_{1}}$ are shown on the right by the dashed and solid lines, respectively. $R_{e}=10^{2}, R_{i}=10^{-4}, k=0.3$ and $\varepsilon=-10^{\circ}$. 


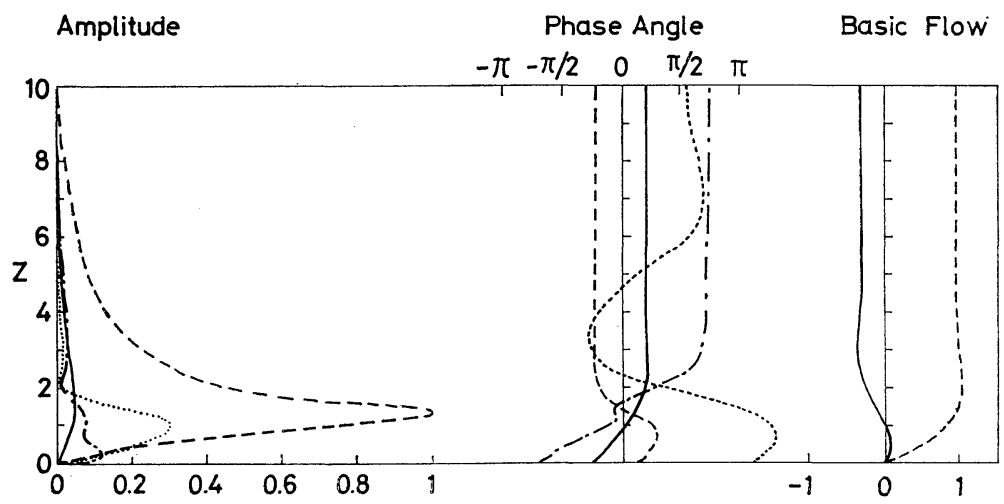

Fig. 10. Same as Fig. 9 except the type II. $R_{e}=10^{3}, R_{i}=10^{-4}, k=0.5$ and $\varepsilon=20^{\circ}$.

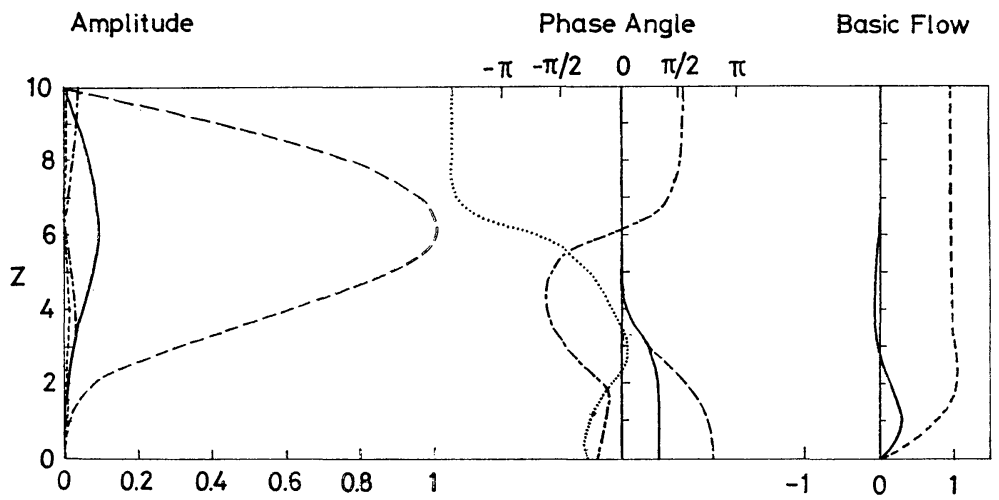

Fig. 11. Same as Fig. 9 except the type III. $R_{e}=10^{2}, R_{i}=10^{-2}, k=1$ and $\varepsilon=0^{\circ}$.

and dashed lines inserted on the right of Figs. 9 11 depict vertical profiles of the velocity components of the Ekman flow perpendicular and parallel to the axis of a perturbation roll, respectively. Figs. 12, 13 and 14 show vertical distributions of the energy conversion associated with the unstable perturbations shown in Figs. 9, 10 and 11 , respectively. The energy conversion terms, $-\left\langle v_{\|}{ }^{\prime} w^{\prime} \frac{d \bar{v}_{\|}}{d z}\right\rangle,-\left\langle v_{\perp}{ }^{\prime} w^{\prime} \frac{d \bar{v}_{\perp}}{d z}\right\rangle, R_{i}\left\langle\theta^{\prime} w^{\prime}\right\rangle$ and $R_{e}{ }^{-1}\left\langle\boldsymbol{v}^{\prime} \cdot \nabla^{2} \boldsymbol{v}^{\prime}\right\rangle$ are denoted by the solid lines, the dashed lines, the dash-dotted lines and the dotted lines respectively.

As is shown in Figs. 9 and 10, the perturbations of the types I and II are confined mostly to the nominal boundary layer in which a significant part of the vertical shear is present. The vertical profiles of the phase angles in Figs. 9 and 10 indicate that $w^{\prime}$ is almost in the opposite phase with $v_{\|}{ }^{\prime}$ in the boundary layer and hence this results in transporting the $v_{i t}$ momentum downward. On the contrary $w^{\prime}$ and $v_{\perp}{ }^{\prime}$ are in phase in the boundary layer so that the $v_{\perp}$-momentum is transported upward. It should be noticed in Figs. 12 and 13 that the resulting energy conversions are from the kinetic energy of the basic flow to that of the perturbations for both the types I and II. These features are quite similar to those of unstable perturbation in the Ekman flow with neutral stratification (Lilly, 1966). Correlations between $w^{\prime}$ and $\theta^{\prime}$ almost vanish except a thin lowest layer and their contributions to energy conversion are so small that the term $\left\langle P, K^{\prime}\right\rangle$ is neglected in each of Figs. 12 and 13.

Figure 11 shows that the amplitude of the perturbation of the type III becomes large above the nominal boundary layer. The phase angle of $w^{\prime}$ is coincident with that of $\theta^{\prime}$ in the upper layer where both of their amplitudes are so 


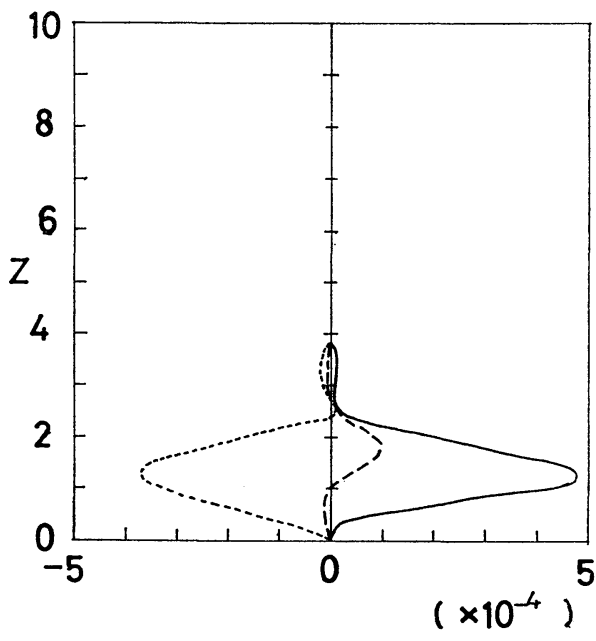

Fig. 12. Vertical distributions of energy conversion terms associated with an unstable perturbation of the type $\mathrm{I}$ adopted in Fig. 9.

$-\left\langle v_{\|}{ }^{\prime} w^{\prime} \frac{d \overline{v_{\|}}}{d z}\right\rangle,-\left\langle v_{\perp}{ }^{\prime} w^{\prime} \frac{d \overline{v_{\perp}}}{d z}\right\rangle$, $R_{i}\left\langle\theta^{\prime} w^{\prime}\right\rangle$ and $R_{e}^{-1}\left\langle\boldsymbol{v}^{\prime} \nabla^{2} \boldsymbol{v}^{\prime}\right\rangle$ are denoted by solid, dashed, dashdotted and dotted lines, respectively. Dash-dotted line is not shown in Figs. 12 and 13 because of small values of $R_{i}\left\langle\theta^{\prime} w^{\prime}\right\rangle$.

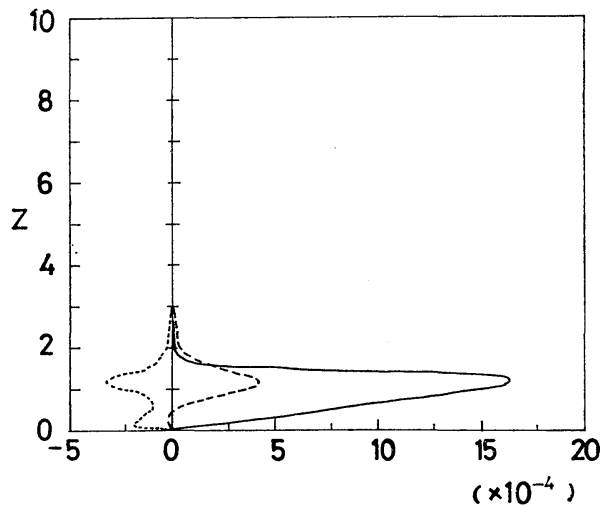

Fig. 13 Same as Fig. 12 except the type II adopted in Fig. 10.

large that conversion of the potential to the kinetic energy is dominant in the energy conversion processes associated with the unstable perturbation of the type III as indicated in Fig. 14. It is further seen in Figs. 11 and 14 that the $v_{i}$-momentum is transported down the vertical gradient of $v_{\|}$and the vertical transport of the

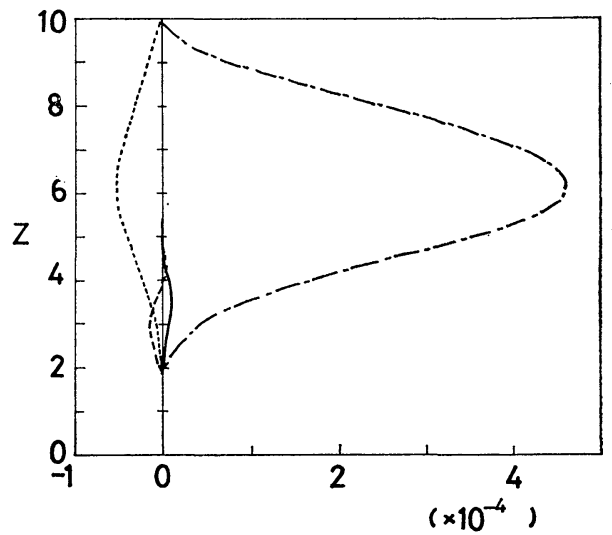

Fig. 14. Same as Fig. 12 except the type III adopted in Fig. 11.

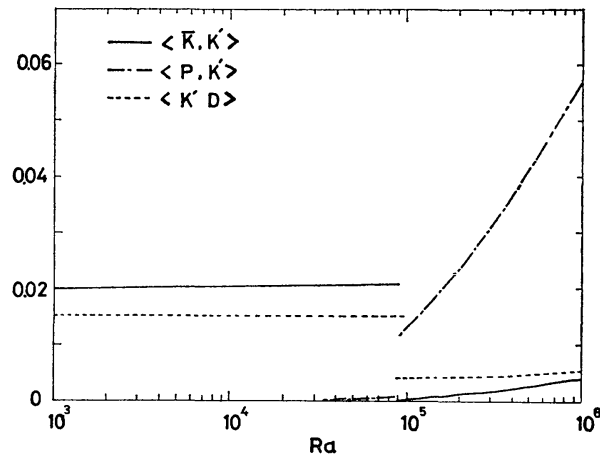

Fig. 15. Energy conversion terms versus the Rayleigh number for $R_{e}=10^{2}, k=$ 0.3 and $\varepsilon=-10^{\circ}$.

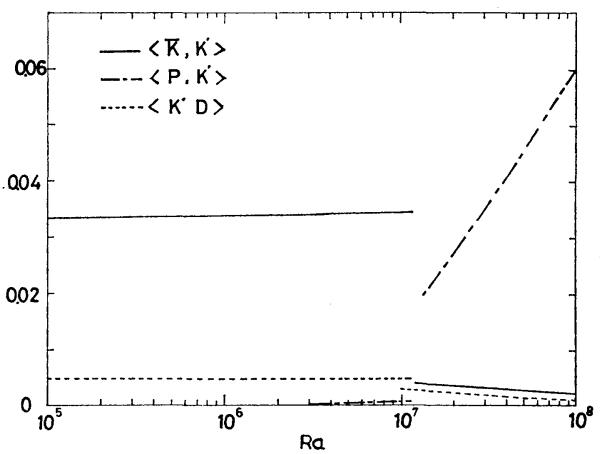

Fig. 16. Same as Fig. 15 except $R_{e}=10^{3}$, $k=0.5$ and $\varepsilon=20^{\circ}$.

$v_{\perp}$-momentum is against the gradient of $\bar{v}_{\perp}$. This feature may be similar to that of thermal instability of a shear flow (Asai, 1970a and b).

Figures 15 and 16 show variations of each quantity of the energy conversion terms normalized 


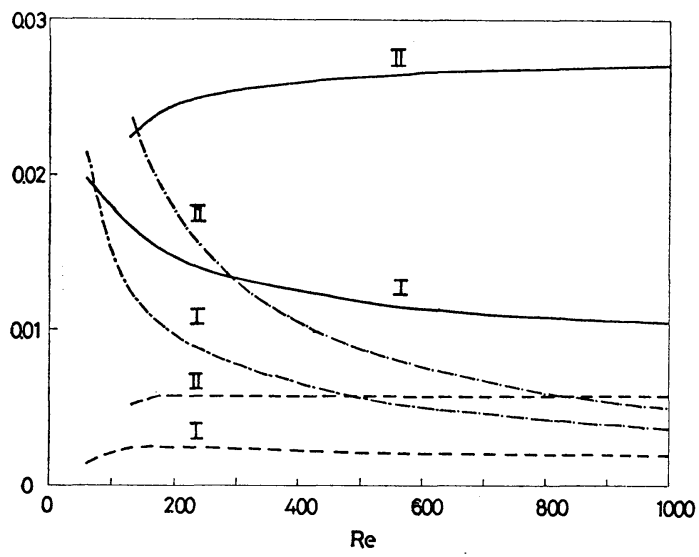

Fig. 17. Energy conversion terms versus the Reynolds number for unstable perturbations of the type I $\left(k=0.3, \varepsilon=-10^{\circ}\right)$ and the type II $\left(k=0.5, \varepsilon=20^{\circ}\right)$, respectively, for $R_{a}=2 \times 10^{4}$.

$-\left\langle v_{\|}^{\prime} w^{\prime} \frac{d \overline{v_{\|}}}{d z}\right\rangle, \quad-\left\langle v_{\perp}^{\prime} w^{\prime} \frac{d \overrightarrow{v_{\perp}}}{d z}\right\rangle$ and $R_{e}^{-1}\left\langle\boldsymbol{v}^{\prime} \cdot \nabla^{2} \boldsymbol{v}^{\prime}\right\rangle$ are denoted by solid, dashed and dash-dotted lines.

by $2\left\langle K^{\prime}\right\rangle$ with $R_{a}$ for the two sets of parameters, (1) $k=0.3, \varepsilon=-10^{\circ}, R_{e}=10^{2}$ and (2) $k=$ $0.5, \varepsilon=20^{\circ}, R_{e}=10^{3}$, respectively. $\left\langle\bar{K}, K^{\prime}>\right.$ is denoted by the solid line, $\left\langle P, K^{\prime}\right\rangle$ by the dash-dotted line and $\left\langle K^{\prime} D\right\rangle$ by the dotted line. A split of each line into two portions suggests the presence of switching unstable perturbations from one type to another. Each term of the energy conversion associated with unstable perturbations shown on the left of Figs. 15 and 16 is slightly dependent on $R_{a}$, while an increase of the $\langle P, K\rangle$-term with $R_{a}$ shown in the right is remarkable. Thus the latter unstable perturbation of the type III which is of thermal origin is separated distinctly from the former two.

Variations of each quantity of the energy conversion terms normalized by $2\left\langle K^{\prime}\right\rangle$ with $R_{e}$ are shown in Fig. 17 for two unstable perturbations for a fixed value of $R_{a}=2 \times 10^{4}$. One is of $k=0.3$ and $\varepsilon=-10^{\circ}$ which is almost a preferred perturbation of the type $I$ and the other is of $k=0.5$ and $\varepsilon=20^{\circ}$ which is a preferred perturbation of the type II. Solid, dashed and dash- dotted lines denote $-\left\langle v_{\|}{ }^{\prime} w^{\prime} \frac{d \bar{v}_{\|}}{d z}\right\rangle,-\left\langle v_{\perp}{ }^{\prime} w^{\prime} \frac{d \bar{v}_{\perp}}{d z}\right\rangle$ and $R_{e}^{-1}\left\langle\boldsymbol{v}^{\prime} \cdot \nabla^{2} \boldsymbol{v}^{\prime}\right\rangle$ respectively. The $\left\langle P, K^{\prime}\right\rangle$-terms are not inserted in Fig. 17 for both the perturbations because of their small values compared with the other terms. As is expected, the $\left\langle K^{\prime} D\right\rangle-$ terms decrease with increasing $R_{e}$ for both the cases. It is striking, however, that the $-\left\langle v_{\|}^{\prime} w^{\prime} \frac{d \bar{v}_{\|}}{d z}\right\rangle-$ term increases with increasing $R_{e}$ for the unstable perturbation of the type II while it reverses for the unstable perturbation of the type I. Therefore it seems that the parallel component of the basic flow to the perturbation roll plays a significant role to destabilize the perturbation of the type I. Following the discussion made by Lilly (1966), the instability of the type I may be called "parallel instability" and the instability of the type II "inflection point instability".

\section{Conclusions}

The stability properties of the Ekman flow in an unstably stratified fluid layer are investigated on the basis of perturbation analysis. The results obtained are summarized as follows.

1. There may appear three types of instabilities different from one another i.e., parallel instability, inflection point instability and thermal instability. The thermal instability, which is of gravitational origin and is modified by the shear flow, is dominant for $R_{i} \gtrsim 10^{-3}$, while the other two, which are of inertial origin and are modified by the thermal stratification, are observed for $R_{i} \lesssim 10^{-3}$. Furthermore the parallel instability may be preferred for $55 \lesssim R_{e} \lesssim 150$ and the inflection point instability predominates for $R_{e} \gtrsim 150$.

2. The preferred unstable perturbation of the thermal instability is characterized by the horizontal scale of about $6 \mathrm{D}$ and moves at the velocity of the geostrophic flow. The perturbation expands throughout an entire layer of unstably stratified fluid and is associated primarily with the upward heat transport through which the potential energy is released to the perturbation kinetic energy.

3. The preferred unstable perturbation of the parallel instability which is of a roll vortex has a wavelength of $21 D$ with an orientation angle of $10^{\circ}$ to the right of the geostrophic flow, whil that of the inflection point instability has a wavelength of $12 \mathrm{D}$ with an orientation angle of $20^{\circ}$ to the left of the geostrophic flow. Both the perturbations move at the velocity components of the Ekman flow perpendicular to the axes of 
the perturbation rolls around their lowest inflection points, respectively. The perturbations whose substantial part is confined in the boundary layer containing significant shear of the flow are characterized by the predominance of conversion processes of kinetic energy of the Ekman flow to that of the perturbations. Hence it should be mentioned that both the parallel and the inflection point instabilities of the Ekman flow are slightly influenced by thermally unstable stratification in the range of $R_{i} \lesssim 10^{-3}$.

\section{Remarks}

The investigation described here was extended to the Ekman flow with an unstable stratification confined to the boundary layer below the height of $\pi D$ and with a neutral one above the underlying unstable layer. The results showed that a critical Richardson number separating the thermal instability from either the parallel instability or the inflection point instability is $\sim 10^{-2}$ which is almost the same as that for the variable shear flow (Asai, 1970b). It was further observed that a preferred perturbation of the type III might have a definite orientation angle as well as a wavenumber. The perturbation moves at the velocity component of the Ekman flow perpendicular to the roll axis around the level at which the perturbation amplitudes of the potential temperature and the vertical velocity attain their maxima. Properties of both the parallel instability and the inflection point instability are slightly influenced by confining thermally unstable stratification to the lower boundary layer.

\section{Acknowledgments}

This work was financially supported by Funds for Scientific Research from the Ministry of Education. The numerical computations were made with the use of a FACOM 230-60 computer at the Data Processing Center of Kyoto University. Thanks are due to Miss $M$. Ōta for drafting the figures and typing the manuscript.

\section{References}

Angell, J.K., 1963: Meteorological applications of constant volume balloons. Meteor. Mag., 92, 3748.

, D.H. Pack and C.R. Dickson, 1968: Lagrangian study of helical circulations in the planetary boundary layer. J. Atmos. Sci., 25,
707-717.

Asai, T. and I. Nakasuji, 1968: A numerical investigation of hydrodynamic instability of a shear flow in a thermally unstable fluid layer. Special Contr. Geophys. Inst., Kyoto Univ., 8, 31-44. , 1970a: Three-dimensional features of thermal convection in a plane Couette flow. J. Meteor. Soc. Japan, 48, 18-29.

, 1970b: Stability of a plane parallel flow with variable vertical shear and unstable stratification. J. Meteor. Soc. Japan, 48, 129-139.

, and I. Nakasuji, 1971: A note on application of finite-difference method to stability analysis of boundary layer flows. Contr. Geophys. Inst., Kyoto Univ., 11, 25-33.

Barcilon, V., 1965: Stability of a non-divergent Ekman layer. Tellus, 17, 53-68.

Brown, W.B., 1961: A stability criterion for threedimensional laminar boundary layers. Boundary Layer and Flow Control, 2, Pergamon Press, New York, N.Y. 913-923.

Brown, R.A., 1970: A secondary flow model for the planetary boundary layer. J. Atmos. Sci., 27, 742-757.

Chandrasekhar, S. and D.D. Elbert, 1955: The instability of a layer of fluid heated below and subject to Coriolis forces. II. Proc. Roy. Soc. London, A 231, 198-210.

Faller, A.J., 1963: An experimental study of the instability of the laminar Ekman boundary layer. J. Fluid Mech., 15, 560-570.

, 1965: Large eddies in the atmospheric boundary layer and their possible role in the formation of cloud rows. J. Atmos. Sci., 22, 176-184.

, and R.E. Kaylor, 1966: A numerical study of the instability of the laminar Ekman boundary layer. J. Atmos. Sci., 23, 466-480.

, 1967: Instability of the Ekman spiral with applications to the planetary boundary layers. Phys. Fluids, S 212-219.

Gregory, N., J.T. Stuart and W.S. Walker, 1955: On the stability of three-dimensional boundary layers with application to the flow due to a rotating disk. Phil. Trans. Roy. Soc. London, A 248, 155-199.

Hanna, S., 1969: The formation of longitudinal sand dunes by large herical eddies in the atmosphere. J. Appl. Meteor., 8, 874-883.

Kaylor, R.E. and A.J. Faller, 1972: Instability of the stratified Ekman boundary layer and the generation of internal waves. J. Atmos. Sci. 29, 497509.

Kuettner, J.P., 1959: The band structure of the atmosphere. Tellus, 11, 267-294.

1971: Cloud bands in the earth's 
atmosphere: Observation and theory. Tellus, 23, 404-425.

Lilly, D.K., 1966: On the stability of Ekman boundary flow. J. Atmos. Sci., 23, 481-494.

Pack, D.H., 1962: Air trajectories and turbulence statistics from weather radar using tetroons and radar transponders. Mon. wea. Rev., 90, 491-506. Plank, V.G., 1966: Wind conditions in situations of patternform and non-patternfrom cumulus convec- tion. Tellus, 18, 1-12.

Tatro, P.R. and E.L. Mollö-Christensen, 1967: Experiments on Ekman layer instability. J. Fluid Mech., 28, 531-544.

Thomas, L.H., 1953: The stability of plane Poiseuille flow. Phys. Rev., 91. 781-783.

Woodcock, A.H., 1940: Convection and soaring over the open ocean. J. Mar. Res., 3, 248-253.

\title{
静力学的に不安定な成層をした Ekman 境界層流の安定性について
}

\author{
浅井富雄・中筋 勲 \\ 京都大学理学部地球物理学教室
}

静力学的に不安定な成層をもつ Ekman 境界層流の安定性を, 摂動論にもとついて考察する. この場合, 流れのシ アーの影響を受けた重力不安定に起因する熱的不安定（対流）と，成層の影響を受けた流れのシアーに起因する慣性 不安定が発現する. 両者の卓越領域は Richardson 数 Riによって区別される.すなわちここで定義された Richardson 数を用いると, 前者は $R_{i} \gtrsim 10^{-3}$ ，後者は $R_{i} \lesssim 10^{-3}$ のときそれぞれ卓越する. 慣性不安定はさらに Reynolds 数 $R e$ によって異なる機構による 2 種類の不安定に分離される。一つは低 Reynolds 数領域, $55 \lesssim R_{e} \lesssim 150$, 他は高 Reynolds 数領域, $R_{e} \gtrsim 150$ で卓越するものである。これら 3 種類の安定性やそれぞれに対応する不安定じょう乱 の特性を摂動方程式系の数値解を求めて詳しく調べる. 慣性不安定の両者とも， $R_{i} \lesssim 10^{-3}$ の領域では不安定成層の 影響をほとんど受けないことが示される。 